\title{
Grand Theft Auto is a "Sandbox" Game, but There are Weapons, Criminals, and Prostitutes in the Sandbox: Response to Ferguson and Donnellan (2017)
}

\author{
Alessandro Gabbiadini $\mathbb{1}^{1} \cdot$ Brad J. Bushman $^{2} \cdot$ Paolo Riva $^{1} \cdot$ Luca Andrighetto $^{3} \cdot$ \\ Chiara Volpato ${ }^{1}$
}

Received: 25 July 2017 / Accepted: 27 July 2017 / Published online: 23 August 2017

(C) Springer Science+Business Media, LLC 2017

\begin{abstract}
In this issue, (Ferguson, C. J., \& Donnellan, B. D., Journal of Youth and and Adolescence, published online 21 June 2017) criticize one of our studies (Gabbiadini, A., Riva, P., Andrighetto, L., Volpato, C., \& Bushman, B. J., PLoS ONE, 11: 1-14, 2016) that found violent sexist video games can reduce empathy for female violence victims in male players who identify with violent male game characters, and do so by increasing masculine beliefs. Their main criticism is a "straw person" argument built on a claim that we never made (i.e., a direct effect of sexist-violent video games on empathy). They also made several other criticisms of our article. We appreciate the opportunity to respond to their criticisms in this article. We also point out some flaws in their reanalysis. Despite their criticisms, the core contributions of our original article remain intact.
\end{abstract}

Keywords Violent video games - Sexist video games · Empathy $\cdot$ Sexism $\cdot$ Child development $\cdot$ Reanalysis

\section{Introduction}

In 2016, we published a study (Gabbiadini et al. 2016) showing that playing the violent-sexist video game Grand Theft Auto (GTA) increases the endorsement of masculine beliefs, especially among male players who highly identify with violent male game characters. We also found that the

\footnotetext{
Alessandro Gabbiadini

ale.gabbiadini@gmail.com

University of Milano-Bicocca, Milan, Italy

2 The Ohio State University, Columbus, OH, USA

3 University of Genova, Genoa, Italy
}

endorsement of masculine beliefs, in turn, reduced the empathy toward female violence victims.

Ferguson and Donnellan (2017) raised some concerns about our article, and reanalyzed our data. They created a "straw person" claim that we never made (i.e., a direct effect of sexist-violent video games on empathy), and then they criticized us based on that claim. They also made seven additional criticisms, which we respond to in this article. First, they question whether GTA is a sexist video game. Second, they criticize our random assignment approach. Third, they argued that gender should not be a moderator. Fourth, they criticize our use of video game violence ratings as a covariate. Fifth, they criticize our measure of masculine beliefs. Sixth, they criticize our measure of identification with game characters. Seventh, they criticize our failure to pre-register our study. We appreciate this opportunity to respond to these criticisms. We believe it can be productive to have an open debate on such issues.

\section{It is Easy to Knock Down a Straw Person}

In their reanalysis, Ferguson and Donnellan (2017) expressed concern about how moderated mediation models are used to support causal inferences. It is crucial to note that conditional process models focus on the conditional nature of an indirect effect. In other words, how an indirect effect is moderated by another variable. As Hayes (2013) notes, "moderated mediation means moderated indirect effects" (p. 387). Interpretive focus in a conditional model is directed at estimating the indirect effect, and how that effect varies as a function of one or more moderators (i.e., levels of individual differences). Specifically, if we conceptualize the indirect effect of an independent variable $(\mathrm{X})$ on a dependent variable ( $\mathrm{Y}$ ) through a mediator (M) as 
depending on the level of a moderator (W), we are assessing how the size of the indirect effect linking $\mathrm{X}$ to $\mathrm{Y}$ through $\mathrm{M}$ differs between groups when increasing of one unit the value of $\mathrm{W}$. In light of these assumptions, we do not see the reanalysis reported by Ferguson and Donnellan (2017) to be particularly relevant, because direct effects are not a necessary condition for indirect effects (see Hayes 2013).

Ferguson and Donnellan (2017) reported that the intent of their reanalysis was to examine the robustness of the claims about media effects made in light of the effects that would be expected by theory. However, we never theorized a direct effect of exposure to sexist videogames on empathy toward female violence victims. Instead, throughout our article we consistently talked about conditional indirect effects of exposure to violent-sexist video games on empathy, which we hypothesized (and found) to emerge through the mediation of masculine beliefs on different levels of identification with the game character, especially for male players (i.e., gender was the moderator). Accordingly, the main analyses presented in Ferguson and Donnellan just confirm what we found and already reported in our original article (i.e., no direct effects of type of video games on empathy).

In their reanalysis, Ferguson and Donnellan (2017) tested a basic model (PROCESS Model 1) in which game condition (the $\mathrm{X}$ variable in the Process Model) led to reduced empathy (Y) moderated by masculine beliefs (M). Indeed, we proposed that the exposure to violent and sexist video games could reinforce masculine beliefs. As discussed in our article, masculine norms are reinforced in GTA video games. Thus, we proposed (and found) that GTA gameplay increased masculine beliefs. Masculine beliefs, in turn, were negatively related to empathy for female violence victims (see Gabbiadini et al. 2016, p. 3). No clear theoretical rationale is given by Ferguson and Donnellan for testing this alternative model. Thus, we have concerns about why they considered masculine beliefs as a moderator. We theorized masculine beliefs to be a mediator rather than a moderator.

Ferguson and Donnellan (2017) also tested a second alternative model in which identification with the game character moderated the effect of type of video game on empathy. In our original article, we hypothesized that the identification with the game character would moderate the effects of the type of video games on masculine beliefsnot on empathy. Thus, we have concerns about this analysis as well.

To test our assumptions in an alternative way, it is possible to run a simple mediation model (PROCESS model 4) with type of video game (i.e., violent-sexist vs. violent or neutral) as the predictor, masculine beliefs as the mediator, and empathy as the outcome. Violent-sexist games increased masculine beliefs $(b=0.21, \mathrm{SE}=0.07, t(152)$
2.85, $p=.005)$, and masculine beliefs were negatively related to empathy for female violence victims $(b=-0.48$, $\mathrm{SE}=0.10, t(151)=-4.69, p<.001)$. As expected, the direct effect of type of video game on empathy was not significant $(p=.29)$, whereas the indirect effect was significant $(b=-0.10,95 \% \mathrm{CI}=-0.22$ to -0.030$)$. The model remained significant even after controlling for participants' age, frequency of video game play, and violence ratings. Adding the covariates, violent-sexist games increased masculine beliefs $(b=0.50, \mathrm{SE}=0.15, t(147)=$ $3.28, p=.001)$, and masculine beliefs were negatively related to empathy for female violence victims $(b=-0.47$, $\mathrm{SE}=0.10, t(146)=-4.34, p<.001)$. The direct effect of type of video games on empathy was not significant $(p$ $=.48)$, whereas the indirect effect was still significant $(b=$ $-0.23,95 \% \mathrm{CI}=-0.49$ to -0.075 ).

Although we agree with Ferguson and Donnellan (2017) that our hypothesized model was rather complex, we believe that the theoretical rationale underlying each aspect of our model was clearly articulated in our article. As already pointed out, we never claimed a direct association between exposure to violent-sexist video games and empathy. Accordingly, the alternative models tested and proposed in Ferguson and Donnellan (2017) could be misleading for readers, as they are not theoretically wellanchored and are distant from our original hypotheses. Indeed, our findings are entirely consistent and sustained by previous research in the field of media effects (see Dill et al. 2008; Dill and Thill 2007; Fox et al. 2013, 2014).

\section{Classification of GTA as a Sexist Game}

In their title, Ferguson and Donnellan (2017) put the word "sexist" in quotation marks, called "scare quotes." Scare quotes are often used to call out nonstandard or unusual terms, or merely to convey irony or skepticism about a term. The term sexist is not a nonstandard or unusual term. The term sexism generally refers to hostility toward females (i.e., hostile affect and negative stereotypes) and the endorsement of traditional gender roles (i.e., confining women to roles accorded less status and power than those of men; see Glick and Fiske 1997). Thus, it is surprising that Ferguson and Donnellan claimed that there is little consensus on what defines a sexist game, and questioned whether GTA was a sexist video game. GTA certainly meets this definition of a sexist game. In almost all chapters of GTA game series, players can pick up female prostitutes and pay to have sex with them. Such behavior is encouraged by tips on many YouTube clips and forums. In fact, players' life points go up when they have sex with a prostitute (see for an example https://www.youtube.com/watch? $\mathrm{v}=$ Tb7HIKThkU - 4:11 min). Then, it is common practice 
among GTA players to kill the prostitute after having sex with her, so they can get their money back. Nevertheless, we do not have to rely on YouTube clips to establish that GTA is a violent-sexist game series. The violent-sexist games that we used in our study, GTA San Andreas and GTA Vice City, are described in the PEGI database (Pan European Game Information) as "suitable for persons aged 18 years and over only". The PEGI webpage also reported that both these video games contain "extreme violence, violence toward defenseless people, sexual violence, and strong language". PEGI is the organization that rates video games for European countries, including Italy where our participants were from. PEGI is similar to the Entertainment Software Rating Board (ESRB) system used in the U.S., which gives GTA similar ratings. Both games are rated $\mathbf{M}$ for Mature players 17 and older. GTA San Andreas contains the following content descriptors: "Blood and Gore, Intense Violence, Strong Language, Strong Sexual Content, Use of Drugs." Similarly, GTA Vice City contains the following content descriptors: "Blood and Gore, Strong Language, Strong Sexual Content, Violence". Thus, it is not just our opinion that the GTA games we used are violent and sexist. They are labelled as such by PEGI and ESRB.

Instead of defining GTA as a sexist video game, Ferguson and Donnellann (2017) defined GTA as a "sandbox game". The terms "sandbox," "open world," and "free roam" are used to describe video games where a player can move freely through a virtual world, rather than in a linear fashion. Yet, all the chapters of GTA develop around a storyline and allows players to take on the role of a criminal who plans to rise through the ranks of organized crime. Players are given various missions that must be completed to progress through the storyline and complete the whole game. For instance, in GTA Vice City, players have to buy a strip club in order to please a criminal boss. By spending a total of $\$ 300$ on the strip-tease dances, the player will complete the club's asset mission. Although GTA can be considered as a "sandbox game," the sandbox is full of prostitutes, poledancers, weapons, and criminals.

\section{Is Playing with Sexist Video Games Just Harmless Fun?}

Our work (see Gabbiadini et al. 2016) is certainly not the first to suggest that violent sexist video games such as GTA can influence the thoughts, feelings, and behaviors of players. In fact, a scientific consensus is beginning to emerge around the potentially harmful effects of sexist violent video games on players. For example, one study found that exposure to images of sex-typed video game characters from GTA (vs. images of professional men and women) increased judgments and attitudes supporting aggression against women (Dill et al. 2008). Another recent study (Fox et al. 2014) found that female participants who played with sexualized characters in a video game reported higher rape myth acceptance scores than participants in other conditions. They also had increased body-related thinking, which can lead to increased self-objectification. The authors suggested that those who use sexualized characters in video games are more likely to develop harsh attitudes toward women and to blame a rape victim for the act (Fox et al. 2014). Another study found that violent video games can be a risk factor for individuals who are already prone to sexually violent fantasy, offering a safe place to 'practice' the activity in ways that feed fantasy and preexisting cognitive scripts (Helfgott 2015).

Modern video games are saturated with stereotypes of women, and these games may cultivate sexism (Bègue et al. 2017). One content analysis found that female characters are far more likely to be portrayed as more sexualized than male characters (60 to 1\%; see Dill and Thill 2007). Female characters often have a huge bust with disproportionally small waist and hips. In addition, female characters tend to play minor roles. These games not only affect the way young women view themselves, they also encourage young men to look at women as sex objects (Dill and Thill 2007). Confirming these findings, another recent study (Lynch et al. 2016) evaluated 571 playable female characters in video games released from 1989 to 2014 and concluded that despite an increase in games featuring playable female characters, games still depict female characters as sexualized and in more minor roles.

\section{Randomization in Real-World Contexts}

Recently, some scholars (e.g., Ferguson 2015) have pointed out that most video game studies rely on college student samples, and that these studies are arguably not illuminating for answering questions about harm to minors, which is the population of primary interest to the general public, parents, and policy makers. It is noteworthy that our study was conducted with minors in a real context (i.e., high school) rather than with college students in a lab context.

As suggested by Ferguson (2015), the adoption of a sample of the population of interest allows researchers to generate evidence with a greater external validity. We agree with this point; however, working in a field setting with adolescents sets some limits over the experimenter's freedom. In our study (Gabbiadini et al. 2016), the internal committee of the high school in which data were collected prevented us from using some items due to ethical reasons (see section on Masculine Beliefs Measure), gave us a 1-h time limit for each experimental session, and gave us only one week to collect all data. Moreover, other practical limits 
related to time management of each classroom at school did not allow us to assign participants to group conditions in a complete randomized way. Instead, we had to randomly assign classrooms to conditions. As suggested by the Editor of PLOS ONE, we discussed this issue on the webpage (http://journals.plos.org/plosone/article/comments?id=10.

1371/journal.pone.0152121). That is why we used participants' age as a covariate in our analyses. Importantly, both in our analyses and in Ferguson and Donnellan reanalysis, participants' age, when entered as covariate, did not affect any of the results.

\section{Gender as a Moderator}

Ferguson and Donnellan (2017) tested a third alternative model (PROCESS Model 7) that excluded gender as a moderator, claiming that such a model would have been theoretically defensible because theories regarding the effects of video games do not typically hypothesize gender differences. However, given that our original article deals with violence toward women, we believed that gender was an important moderator. Considering the gendered phenomenon under investigation, our original work reported our expectations of different effects for males and females. It is hard to understand how collapsing males and females together could be considered as an appropriate analytical strategy for our data set.

\section{Video Game Violence as Covariate}

Ferguson and Donnellan (2017) said it was unclear why video game violence ratings should be used as a covariate. As we reported in our original article (Gabbiadini et al. 2016; p. 6), post-hoc comparisons indicated that the violentsexist games had higher violence ratings than the violenceonly games. Because it is crucial to disentangle violent content from sexual content, we included the violence ratings as a covariate (for a similar approach, see Bushman and Anderson 2002; Anderson and Carnagey 2009; Barlett and Rodeheffer 2009).

\section{Masculine Beliefs Measure}

The high school internal committee imposed a number of limitations on the wording of items from the Male Role Norms Inventory (MRNI; Levant et al. 2010). The MRNI assesses seven theoretically derived traditional norms: (1) Avoidance of Femininity, (2) Fear and Hatred of Homosexuals, (3) Self-Reliance, (4) Aggression, (5) Achievement/Status, (6) Non-relational Attitudes toward Sexuality, and (7) Restrictive Emotionality. It also includes a Nontraditional Attitudes toward Masculinity scale, which includes items that reflect violations of traditional male norms. After a careful analysis, the committee asked us to adapt or to drop some items that were considered too explicit for minors, considering that some of the participants were as young as 15 years old (i.e., "Men should always take the initiative when it comes to sex").

Moreover, the maximum available length of our experimental session to one hour further limited the number of items we could include in the original questionnaire. Given these restrictions, we selected a pool of five subscales from the original MRNI and considered three items for each subscale (i.e., Self-Reliance, Aggression, Achievement/ Status, Non-relational Attitudes toward Sexuality and Restrictive Emotionality).

When selecting the items, we chose items more appropriate for minors. The wording of items was also adapted to our sample. For instance, the original item "Men should always take the initiative when it comes to sex" was adapted as "Guys should always take the initiative when it comes to love relationships". All the 15 selected items were adapted, except for the items composing the self-reliance subscale, which accidentally were reported as they are in the original scale. Thus, these items were excluded from the analyses (see note 3 in Ferguson and Donnellan 2017). Although our article should have contained a more detailed discussion of the items we selected and changes, there were valid reasons for making these selections and changes.

Nevertheless, given the concerns raised by Ferguson and Donnellan (2017, see note 3), we performed additional analyses considering all the 15 items collected for the masculine beliefs scale (Cronbach $\alpha=.79$ ). Thus, we considered a conditional process model (PROCESS model 11) in which the type of video game was entered as the predictor, gender, and the identification with the game character as the moderators, masculine beliefs (computed considering all the 15 available items) as the mediator, and empathy toward female violence victims as the outcome. As in the original model, participants' age, video game violence rating, and frequency of video game play were included as covariates. The predicted 3-way interaction between type of video game played, participants' gender, and identification with the game character on masculine beliefs was significant $(b=.24, \mathrm{SE}=.10, t(140)=2.35, p=.020 ; 95 \%$ $\mathrm{CI}=0.039$ to 0.45 ). Thus, our finding was robust to how masculine beliefs were operationally defined.

\section{Identification with the Game Character}

Adolescents seem more likely than adults to identify with their avatars (Blinka 2008), and in our study we assumed 
the identification with the game character as one possible mechanism for better understand the process leading from violent sexist games play to reduced empathy for female violence victims. In order to assess the individual level of identification with the game character, we adapted the scale proposed by Van Looy et al. (2012), which is composed of three subscales: (1) Wishful Identification, (2) Similarity Identification, and (3) Embodied Presence.

To keep our study within the 1-h time limit given to us by the high school, we dropped the "Similarity Identification" subscale, which is defined as "the degree to which the player sees their avatar as similar to him/herself" (Van Looy et al. 2012, p.129). This subscale was considered less relevant because it is typically used for MMORPG (Massively Multiplayer Online Role Playing Games) virtual environments rather than stand-alone games like the ones we used (Van Looy et al. 2012). Because the reliability for the five-item wishful identification subscale was rather low (Cronbach $\alpha=.67$ ), we also decided to drop it from our analyses and to consider only the embodied presence subscale that was much more reliable (Cronbach $\alpha=.92$ ). Theoretically, it is also a better measure. Indeed, embodiment creates a powerful and very realistic experience for the players and it represents the strongest mechanism for identification with a virtual representation (Biocca 1997; Klimmt et al. 2010). Such a detailed procedure could have been mentioned in the original article and indeed, it was subsequently amended through a specific comment made in accordance with the PLOS ONE editor on the website of the journal (http://journals.plos.org/plosone/article/comments? $\mathrm{id}=10.1371$ /journal.pone.0152121).

Furthermore, Van Looy and colleagues (2012), established the nomological validity of their proposed scale. Nomological validity has an external orientation, referring to the degree to which constructs accurately predict other constructs, within a shared theoretical model. After validating the avatar identification scale, Van Looy et al. also assessed separate measures for the Empathy toward the avatar and for the Proteus effect (see Tables 4 and 5). The four items present in our published data set (labeled as avatar_id_char_empathyXX), were derived from a 4-item scale used in Van Looy et al. (2012) and adapted to the context of video games (Cohen 2001; Davis 1980).

Following the rationale proposed by Van Looy et al. (2012), we ran a series of analyses considering identification with the game character as the predictor and empathy toward the game character as the outcome. Identification with the game character significantly predicted the empathy toward the game character $\beta=.40, t(152)=5.35, p<.001$, even when controlling for participants' age, violence rating, and frequency of game play. A second analysis, revealed that the identification with the character-computed as the mean of the items for embodied presence and wishful identification-still significantly predicted empathy toward the game character, $\beta=.50, t(152)=7.06, p<.001$, even when controlling for participants' age, violence rating, and frequency of game play.

To support our conclusions and following the theoretical rationale explained in our original article, we modified our proposed model by including a composite index for identification with the game character, computed as the average of the embodied presence 6-item subscale plus the 5-item wishful identification subscale. Thus, conditional process model by using the PROCESS macro Model 11 for SPSS with 5000 bootstrapping samples was computed. In this model, the type of video game was entered as the predictor, gender and the identification with the game character as the moderators, masculine beliefs (15 items) as the mediator, and empathy toward female violence victims as the outcome. As in the original model, participants' age, video game violence rating, and frequency of video game play were also included as covariates. Analyses confirmed the predicted 3-way interaction between type of video game, participants' gender, and identification with the game character on masculine beliefs, even when considering all the available set of items for the avatar identification construct $(b=0.27, \mathrm{SE}=0.11, t(140)=2.46, p=.015 ; 95 \%$ $\mathrm{CI}=0.055$ to 0.50 ). Thus, the three-way interaction we reported in the original article appears to be robust and insensitive to how identification with the game character is measured. This boosts confidence in the reliability of the three-way interaction.

Even though the empathy toward the game character should not be included in the avatar identification construct and scale (see Van Looy et al. 2012), Ferguson and Donnellan (2017) included it. Surprisingly, they sometimes computed identification with the game character as the mean of wishful identification, embodied presence, and empathy toward the character. Theoretically, identification with the game character is not the same as empathy for the game character and should therefore not be combined.

\section{Pre-Registration of Studies}

Psychological science, along with other scientific disciplines, has begun to adopt practices aimed at reducing the frequency of publishing poor quality research. We agree with Ferguson and Donnellan (2017) that more and more studies would benefit from pre-registration, with the aim of preventing publication bias and practices that can increase the likelihood of making Type I errors (Gonzales and Cunningham 2015). However, we are not sure why Ferguson and Donnellan targeted only our article as an example of failure to pre-register a study. Researchers have only recently begun to pre-register studies. For example, Dr. 
Ferguson seems to have started pre-registering some of his studies in 2017, whereas Dr. Donnellan seems to have started pre-registering his studies in 2016. Our data were collected in 2014. Thus, it seems unfair for the them to criticize us for doing something that they perhaps were not doing themselves at the time our data were collected.

\section{Conclusion}

The implications of the Ferguson and Donnellan (2017) reanalysis did not significantly differ from those reported in our original article. Both analyses highlighted the lack of a direct effect of violent-sexist video games playing on empathy. Our original study carefully avoided making such a claim. Furthermore, in our original article, we have never suggested that exposure to violent sexist video games is a necessary or a sufficient cause of decreased empathy for female violence victims. To our knowledge, no mediaviolence researcher has ever made such a claim.

As researchers in the field of media effects, we strongly believe that an open and constructive debate on the effects of violent and sexist video games can contribute to the improvement of research in this area. Modern video games present hyper-realistic environments, complex plots, structured narratives, and immersive virtual world. Because of this complexity, researchers in the media field need to dig deeper into the understanding of underlying processes and mechanisms underlying exposure to modern video games. Human thought, emotion, and behavior is often complex, and complex models are therefore required to understand this complexity.

The effect of violent video games exposure on adolescent development continues to be urgent. We believe that more research is needed to understand how video games affect youth. It is therefore important to identify factors-including media exposure - that, singly and together, may influence how youth view the world and others.

Author Contributions All the authors contributed to the conceptualization, data analysis and writing of the final draft manuscript. All the authors read and approved the final draft of this manuscript.

Compliance with Ethical Standards All procedures described within were developed to comport with APA standards for ethical human participant research.

Conflict of Interest The authors declare that they have no competing interests.

Ethical Approval The original procedures described within received local ethical approval as described in Gabbiadini et al. (2016).
Informed Consent The original procedures described within were conducted with informed consent provided to participants as described in Gabbiadini et al. (2016).

\section{References}

Anderson, C., \& Carnagey, N. L. (2009). Causal effects of violent sports video games on aggression: Is it competitiveness or violent content? Journal of Experimental Social Psychology, 45(4), 731-739.

Barlett, C. P., \& Rodeheffer, C. (2009). Effects of realism on extended violent and nonviolent video game play on aggressive thoughts, feelings, and physiological arousal. Aggressive Behavior, 35(3), 213-224.

Bègue, L., Sarda, E., Gentile, D. A., Bry, C., \& Roché, S. (2017). Video games exposure and sexism in a representative sample of adolescents. Frontiers in Psychology, 8, article 466.

Biocca, F. (1997). The Cyborg's dilemma: Progressive embodiment in virtual environments. Journal of Computer-Mediated Communication, 3(2).

Blinka, L. (2008). The relationship of players to their avatars in MMORPGs: Differences between adolescents, emerging adults and adults. Cyberpsychology: Journal of Psychosocial Research on Cyberspace, 2(1), article 5.

Bushman, B. J., \& Anderson, C. A. (2002). Violent video games and hostile expectations: A test of the general aggression model. Personality and Social Psychology Bulletin, 28(12), 1679-1689.

Cohen, J. (2001). Defining identification: A theoretical look at the identification of audiences with media characters. Mass Communication \& Society, 4(3), 245-264.

Davis, M. (1980). A multidimensional approach to individual differences in empathy. JSAS Catalog of Selected Documents in Psychology, 10(4), 85.

Dill, K., Brown, B., \& Collins, M. (2008). Effects of exposure to sexstereotyped video game characters on tolerance of sexual harassment. Journal of Experimental Social Psychology, 44(5), $1402-1408$.

Dill, K., \& Thill, K. (2007). Video game characters and the socialization of gender roles: Young people's perceptions mirror sexist media depictions. Sex Roles, 57(11), 851-864.

Ferguson, C. J. (2015). Do angry birds make for angry children? A Meta-Analysis of video game influences on children's and adolescents' aggression, mental health, prosocial behavior, and academic performance. Perspectives on Psychological Science, 10 (5), 646-666.

Ferguson, C. J., \& Donnellan, B. D. (2017). Are associations between "sexist" video games and decreased empathy toward women robust? A reanalysis of Gabbiadini et al. 2016. Journal of Youth and and Adolescence, published online 21 June 2017.

Fox, J., Bailenson, J. N., \& Tricase, L. (2013). The embodiment of sexualized virtual selves: The Proteus effect and experiences of self-objectification via avatars. Computers in Human Behavior, 29(3), 930-938.

Fox, J., Ralston, R. A., Cooper, C. K., \& Jones, K. A. (2014). Sexualized avatars lead to women's self-objectification and acceptance of rape myths. Psychology of Women Quarterly, 39(3), 349-362.

Gabbiadini, A., Riva, P., Andrighetto, L., Volpato, C., \& Bushman, B. J. (2016). Acting like a tough guy: Violent-sexist video games, identification with game characters, masculine beliefs, and empathy for female violence victims. PLOS ONE, 11(4), 1-14. 
Glick, P., \& Fiske, S. T. (1997). Hostile and benevolent sexism: measuring ambivalent sexist attitudes toward women. Psychology of Women Quarterly, 21(1), 119-135.

Gonzales, J. E. \& Cunningham, C. A. (2015). The promise of preregistration in psychological research. Psychological Science Agenda, August, 2015. Resource document. www.apa.org/science/about/psa/2014/12/data-sharing.aspx. Accessed 22 July 2017.

Hayes, A. F. (2013). Introduction to mediation, moderation, and conditional process analysis. New York: The Guilford Press.

Helfgott, J. B. (2015). Criminal behavior and the copycat effect: Literature review and theoretical framework for empirical investigation. Aggression and Violent Behavior, 22, 46-64.

Klimmt, C., Hefner, D., Vorderer, P., Roth, C., \& Blake, C. (2010). Identification with video game characters as automatic shift of self-perceptions. Media Psychology, 13(4), 323-338.

Levant, R. F., Rankin, T. J., Williams, C. M., Hasan, N. T., \& Smalley, K. B. (2010). Evaluation of the factor structure and construct validity of scores on the male role norms inventory revised (MRNI-R). Psychology of Men \& Masculinity, 11(1), 25-37.

Lynch, T., Tompkins, J. E., van Driel, I. I., \& Fritz, N. (2016). Sexy, strong, and secondary: A content analysis of female characters in video games across 31 years. Journal of Communication, 66(4), 564-584.

Van Looy, J., Courtois, C., De Vocht, M., \& De Marez, L. (2012). Player identification in online games: validation of a scale for measuring identification in MMOGs. Media Psychology, 15(2), 197-221.

Alessandro Gabbiadini received his $\mathrm{PhD}$ in Quality of life in the Information Society at the University of Milano-Bicocca in 2012. He is now a post-doctoral research fellow at the University of MilanoBicocca. His main research interests focus on morality and violent videogames, social identity in virtual environments, new technologies and social interaction.

Brad J. Bushman is full-professor of communication and psychology at The Ohio State University. He holds the Margaret Hall and Robert Randal Rinehart Chair of Mass Communication. He studies the causes, consequences, and solutions to the problem of human aggression and violence.

Paolo Riva is an assistant professor at University of Milano-Bicocca. His research interests lie broadly in social influence processes with a specific focus on social exclusion. Specifically, he examines the consequences of exclusion and the possible strategies that can buffer against and reduce its effects. In a related vein, he is also interested in the neuromodulation of emotion regulation processes.

Luca Andrighetto received his $\mathrm{PhD}$ in Social Psychology from the University of Padova in 2008 and he was then a post-doctoral research fellow at the University of Milano-Bicocca. Currently, he is Associate Professor at the University of Genova. His work centers around issues of social relations and prejudice, with a focus on intergroup and interpersonal attributions of humanity. Currently, he is also exploring real-life consequences of video-game exposure. He is also Editor of InMind Italy.

Chiara Volpato is full-professor of Social Psychology at the university of Milano-Bicocca. Her main research interests focus on intergroup relationships, psychosocial analysis of historical texts, collective emotions and colonialism, sexism and dehumanization. 